\title{
Pinholes Meet Fabry-Pérot: Perfect and Imperfect Transmission of Waves through Small Apertures
}

\author{
R. Merlin \\ C-PHOM and Department of Physics, University of Michigan, Ann Arbor, Michigan 48109-1040, USA
}

(Received 29 March 2012; published 5 September 2012)

\begin{abstract}
Waves, of wavelength $\lambda$, transmit poorly through apertures of dimensions $\ell \ll \lambda$. Here it is shown that coupling of a subwavelength aperture to an electromagnetic oscillator makes it possible for a focused, diffraction-limited beam that impinges on the aperture to undergo perfect transmission. Ignoring nonradiative losses, and for apertures with closed boundaries in a metallic screen, the transmitted power at the oscillator's natural frequency is enhanced by a factor of $(\lambda / \ell)^{6}$ compared with the nonresonant case. As a nontrivial extension to apertures with open boundaries, an analytically solvable problem is introduced and analyzed, which involves a pair of arbitrarily small slits in a two-dimensional waveguide. The system displays perfect transmission at a frequency corresponding to that of a quasilocalized, cavitylike mode bound to the slits, the frequency of which is below that of the cutoff mode of the continuum. In contrast, and remarkably, the Fabry-Pérot-like resonance with the extended cutoff mode leads to imperfect transmission, comparable to that of an individual, nonresonated slit. An explanation of this single-slitlike behavior is presented, which also applies to the closely related phenomenon of light funneling concerning transmission through subwavelength channels [see F. Pardo et al., Light Funneling Mechanism Explained by Magnetoelectric Interference, Phys. Rev. Lett. 107, 093902 (2011), and references therein].
\end{abstract}

DOI: 10.1103/PhysRevX.2.031015

\section{INTRODUCTION}

It has been known for a long time that small holes, of dimensions $\ell$, are ill suited for transmitting electromagnetic, acoustic, or other disturbances of wavelength $\lambda \gg \ell$. The normalized transmittance $T_{N}$ defined as the ratio between the power transmitted and that incident upon the hole, is at best of order $\ell^{4} / \lambda^{4}[1,2]$. More recently, Ebbesen et al. [3] showed that periodic arrays of small apertures in a metallic film can lead to an extraordinary enhancement of the optical transmission, resulting from the excitation of particular modes of the metallic structure [4-6]. This important discovery has led to numerous ideas for applications in areas such as sensing, near-field microscopy, and light harvesting, that can benefit from the concurrent enhancement of the electric field in the vicinity of the apertures [7].

The mechanisms underlying extraordinary transmission for hole arrays are fairly well understood. In particular, for electromagnetic waves, the distinct and cooperative roles played by surface modes (plasmon polaritons) and waveguide or Fabry-Pérot-type resonances are now well established [6]. The same cannot be said for single apertures. While the utilization of geometric and plasmon resonances to enhance the transmission of small, isolated openings has been considered before [8-16], a unified physical picture

Published by the American Physical Society under the terms of the Creative Commons Attribution 3.0 License. Further distribution of this work must maintain attribution to the author(s) and the published article's title, journal citation, and DOI.
Subject Areas: Metamaterials, Nanophysics, Photonics

tying the various approaches together has not yet emerged. Moreover, the distinction between single and multiple apertures has not been fully appreciated (e.g., a dense array of grooves give perfect transmission [4], whereas a single groove does not [11]). Here, we provide an integrated framework for understanding resonant transmission in two and three dimensions and uncover significant differences in the behavior of localized- and extended-mode resonances [17]. We do so by first introducing a simple yet comprehensive model of a closed-curve aperture coupled to an electromagnetic oscillator that gives perfect transmission, that is, $T_{N} \sim \lambda^{2} / \ell^{2}$, in the absence of all but radiative losses. The model draws from ideas that have been hinted at but not fully treated in the engineering literature $[9,10]$. It applies to openings in resonant cavities [10] and aperture-induced localized states in waveguides, as well as to approaches involving $L C$ [9] and other geometric resonances $[13,16]$ for which the resonant wavelength decreases with the size of the aperture. Next, we extend these ideas to open-boundary apertures by specifically considering transmission through a pair of pinholes in a two-dimensional waveguide. We provide an analytical solution to this problem, which reveals the existence of a localized mode bound to the pinholes whose frequency is below that of the onset of the extended-mode continuum. Resonance between the incident wave and the localized mode leads to perfect transmission. Finally, we show that this problem is related to the well-studied single-slit funneling, that is, extraordinary transmission of waves through subwavelength channels $[8,11,15]$. As in funneling, the key resonance is of the Fabry-Pérot type. However, unlike 
funneling, the transmittance does not decrease with decreasing slit width but exactly matches the incident power for arbitrarily small hole sizes. In funneling and related cases, we find that resonant coupling to waveguide modes and, in particular, to the cutoff mode gives imperfect transmission, with transmitted powers that are on the order of those for single slits off resonance.

\section{RESULTS AND DISCUSSION}

\section{A. Subwavelength aperture coupled to an oscillator}

Consider a small aperture defined by a closed boundary in a conductive wall, and a resonant system, with natural frequency $\Omega$, to which the aperture is coupled. Focusing on electromagnetic waves, we take $\mathbf{m}$ to be the induced, effective magnetic dipole of the opening and $\mathbf{H}_{0}$ to be the tangential component of the time-harmonic magnetic field that would exist in the absence of the opening (similar considerations apply to the effective electric dipole, which arises when the boundary problem involves the normal component of the electric field) $[1,18]$. We assume that the magnetic polarizability tensor is diagonal, and that the only effect of the coupling is to introduce poles at $\omega \approx \pm \Omega$ so that

$$
m=\frac{\Omega^{2} \ell^{3} H_{0}}{\omega^{2}-\Omega^{2}-i \omega / \tau} .
$$

Here $\ell$ is a characteristic length of the aperture, $\omega=$ $2 \pi c / \lambda$ is the angular frequency ( $c$ is the speed of light), and $\tau<\infty$ accounts for losses; a time dependence $e^{-i \omega t}$ is understood throughout. In the absence of other sources of dissipation, the lifetime $\tau$ can be obtained by equating the power delivered by the magnetic field, $P_{D}(\omega)=$ $\operatorname{Re}\left(i \omega m^{*} H_{0}\right) / 2$ to that radiated by the dipole, namely, $P_{R}(\omega)=\omega^{4}|m|^{2} / 3 \pi c^{3}$.

Consistent with the general theory of small apertures [1], Eq. (1) gives $m \approx-\ell^{3} H_{0}$ for $\omega \ll \Omega$ and, therefore, $P_{R} \propto$ $\ell^{6} / \lambda^{4}$. We then recover the well-known off-resonance expression $T_{N} \sim \ell^{4} / \lambda^{4}$. On the other hand, for $\mid \omega^{2}-$ $\Omega^{2} \mid \ll \omega / \tau$, we have $|m| \approx \Omega \tau \ell^{3} H_{0}$ and $\tau=$ $3 \pi c^{3} / 2 \Omega^{4} \ell^{3}$. Using these values, the radiated power is

$$
P_{R}(\Omega)=3 c \lambda^{2} H_{0}^{2} / 16 \pi,
$$

which is comparable to that of an incident beam focused down to a spot of approximately $\lambda$ in size. Thus, we obtain the anticipated result, $T_{N}(\Omega) \sim \lambda^{2} / \ell^{2}$, setting a fundamental bound to the transmittance [19]. As suggested by a straightforward application of Babinet's principle, it is clear that the transmission enhancement and the enhanced cross section of resonant antennas (approximately $\lambda^{2}$; see, e.g., [20]), are closely related phenomena. Just as for antennas, the ideal $(\lambda / \ell)^{6}$ enhancement over the value for $\omega \ll \Omega$ will be significantly reduced in real metals due to conduction losses. The analysis leading to Eq. (2) accounts for the interaction of openings with single plasmons and geometric resonances and, with few changes, for transmission through a pair of holes in resonant cavities [10]. However, as discussed later, Eq. (2) does not apply to single-slit funneling $[8,11,15]$ or other cases involving extended, waveguide modes that do not interact directly with the external field. It is also interesting to point out that $|m|$ doubles its value and, thus, that $P_{R}$ increases by a factor of 4 , if the aperture radiates into half space. In such cases, the reflected beam of a tightly focused wave would change its phase by a factor of $\pi$.

\section{B. Transmission through a slotted parallel-plate waveguide}

The above arguments can be extended to apertures bound by an open curve. Consider wave transmission for the slotted parallel plates shown in Fig. $1 ; h$ is the plate separation and $\Delta$ is the half-width of the slits. The equation to solve is the two-dimensional Helmholtz equation:

$$
\left(\frac{\partial^{2}}{\partial x^{2}}+\frac{\partial^{2}}{\partial z^{2}}+k^{2}\right) \Phi=0
$$

Physically, $\Phi$ represents either one of the Cartesian components of the fields in Maxwell's equations or the freespace quantum wave function in Schrödinger's equation; $k=2 \pi / \lambda$ where $\lambda$ is the vacuum or the de Broglie wavelength. We note that perfect transmission in two dimensions requires that $T_{N} \sim \lambda / \Delta$. In the following and for definitiveness, we concentrate on the electromagnetic problem and assume that the waveguide walls are infinitesimally thin and made of a perfect conductor so that the boundary condition at the walls is $\Phi \equiv 0$ for the component(s) of the magnetic (electric) field perpendicular (parallel) to the plates. We further take $\Phi$ to embody the

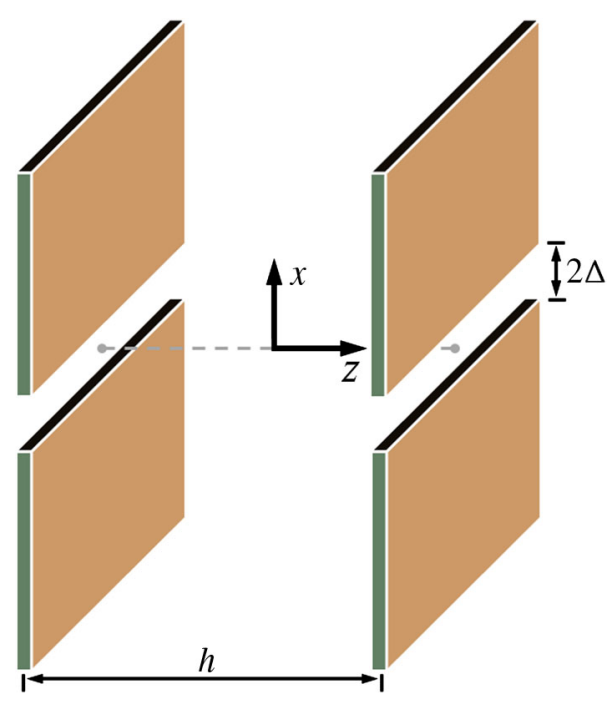

FIG. 1. Geometry of the problem. Slits, defined by $z= \pm h / 2$, $|x|<\Delta$, have been cut in the infinitesimally thin walls of a parallel-plate waveguide. Empty space fills the regions $|z| \geq h / 2$ and between the slotted screens. $E_{y} \equiv 0$ at the walls. 
$y$ component of the electric field $E_{y}$. Thus, following the convention used in recent papers [11,15,21], the geometry is that of transverse-electric (TE) excitation in which the magnetic field is perpendicular to the slit axis. Using results from [22], it can be shown that the transverse-magnetic (TM) case (magnetic field along y) does not give perfect transmission. Concerning the labels TE and TM, we note the unfortunate fact that the current usage of these terms is a transposition of that in the early engineering literature $[8,22,23]$.
Let $Y=Y_{0} J_{1}(k \tilde{\rho}) \sin \tilde{\varphi}$ be the solution to Eq. (3) in the absence of the slits, where $Y_{0}$ is a constant, $\tilde{\rho}^{2}=x^{2}+$ $(z-h / 2)^{2}$, and $\sin \tilde{\varphi}=(z-h / 2) / \tilde{\rho}$. $Y$ is a cylindrical electric field that contains both the incident and reflected waves, with diffraction-limited focus on the right plate. Defining

$$
\kappa(q)= \begin{cases}+\sqrt{k^{2}-q^{2}}, & |q| \leq k \\ +i \sqrt{q^{2}-k^{2}}, & |q|>k,\end{cases}
$$

we write the solution as

$$
E_{y}(x, z)= \begin{cases}\int_{-\infty}^{+\infty} F^{(+)}(q) e^{+i \kappa(z-h / 2)} e^{i q x} d q+\Upsilon & (z>h / 2) \\ \int_{-\infty}^{+\infty}\left(A^{(e)}(q) \frac{\cos \kappa z}{\cos \kappa h / 2}+A^{(o)}(q) \frac{\sin \kappa z}{\sin \kappa h / 2}\right) e^{i q x} d q & (|z|<h / 2) \\ \int_{-\infty}^{+\infty} F^{(-)}(q) e^{-i \kappa(z+h / 2)} e^{i q x} d q & (z<-h / 2)\end{cases}
$$

Here, $A^{(e)}=\left(F^{(+)}+F^{(-)}\right) / 2$ and $A^{(o)}=\left(F^{(+)}-F^{(-)}\right) / 2$ so that both the electric field and the $z$ component of the magnetic field $H_{z} \propto \partial E_{y} / \partial x$ are continuous at the openings.

As an intermediate step to solve our problem, we review briefly the analysis for diffraction by a single slit [23]. Taking $h=0$, and using

$$
F(q)=\frac{1}{2 \pi} \int_{-\Delta}^{+\Delta} E_{y}\left(x^{\prime}, 0\right) e^{-i q x^{\prime}} d x^{\prime},
$$

where $F(q)=F^{( \pm)}(q)$, the continuity of $H_{x} \propto \partial E_{y} / \partial z$ gives

$$
\frac{k Y_{0}}{2}=-i k^{2}\left(1+\frac{1}{k^{2}} \frac{d^{2}}{d x^{2}}\right) \int_{-\Delta}^{+\Delta} E_{y}\left(x^{\prime}, 0\right) H_{0}^{(1)}\left(k\left|x^{\prime}-x\right|\right) d x^{\prime},
$$

where $H_{0}^{(1)}$ is a Hankel function. Recalling the expansion valid for $k \Delta \ll 1$,

$$
H_{0}^{(1)}(k \Delta) \approx i \frac{2}{\pi} \ln (\gamma k \Delta / 2)
$$

$(\gamma=1.78108 \ldots)$ and the following identity from Hilbert transform theory [23]:

$$
\frac{d^{2}}{d \xi^{2}} \int_{-1}^{+1} \sqrt{1-\xi^{\prime 2}} \ln \left|\xi-\xi^{\prime}\right| d \xi^{\prime}=\pi,
$$

we get the following for slits that are narrow relative to the wavelength:

$$
E_{y}(x, 0)= \begin{cases}E_{0} \sqrt{1-x^{2} / \Delta^{2}} & |x|<\Delta \\ 0 & |x|>\Delta\end{cases}
$$

and, therefore,

$$
F(q)=\frac{E_{0} \Delta}{2} \frac{J_{1}(q \Delta)}{q \Delta}
$$

where

$$
E_{0} \approx \frac{\Upsilon_{0}}{2 k \Delta}\left[\ln (\gamma k \Delta / 2)+\frac{2}{k^{2} \Delta^{2}}\right]^{-1} .
$$

Since, to lowest order, $E_{0} \propto \Delta$, it can be shown that the power transmitted is proportional to $\Delta^{4}$. It then follows that $T_{N} \sim \Delta^{3} / \lambda^{3}$. A similar procedure gives $T_{N} \propto$ $(\lambda / \Delta) \ln ^{-2}(\Delta / \lambda)$ for the TM geometry. The difference between the two cases reflects the fact that the effective magnetic dipole density for the TM and TE modes are proportional, respectively, to $1 / \ln \Delta$ (dipole along $y$ ) and $\Delta^{2}$ (dipole along $x$ ) [23]. The appearance of a logarithmic term in the polarizability is a feature of two-dimensional problems. For closed boundaries, all the terms in the magnetic and electric polarizability tensor are proportional to $\ell^{3}[1,18]$.

Coming back to the slotted parallel-plate problem, one can invoke the method of moments [24] to prove that, in the limit $\Delta \ll h$, and for source fields that vary slowly in the scale of the slit width, the approximate solution for $|z|=h / 2$ must be also of the form (5). That is,

$$
F^{( \pm)}(q)=\Gamma^{( \pm)} \frac{J_{1}(q \Delta)}{q \Delta} .
$$

The condition that $\partial E_{y} / \partial z$ be continuous at $z= \pm h / 2$ determines the constant factors $\Gamma^{( \pm)}$.

Equation (4) exhibits singularities at $q=0$ for $k h=p \pi$ (integer $p>0$ ) reflecting the cutoff frequencies of the waveguide or, alternatively, the Fabry-Pérot resonances of the parallel plates. In the vicinity of the lowest cutoff frequency $\omega_{C}=\pi c / h$, we write $k h=\pi-\varsigma(|\varsigma| \ll 1)$ and get

$$
\Gamma^{(+)}=\frac{A}{B^{2}-A^{2}} k \Upsilon_{0}, \quad \Gamma^{(-)}=\frac{-B}{B^{2}-A^{2}} k \Upsilon_{0} .
$$


Here $A=B-C+i \pi k^{2} / 2$ with

$$
\begin{aligned}
B & \approx \frac{1}{2} \int_{|q|<k} \sqrt{k^{2}-q^{2}} \tan \left[\left(\sqrt{k^{2}-q^{2}}\right) h / 2\right] d q \\
& \approx k^{2} \sqrt{\frac{2 \pi}{|\varsigma|}} \times \begin{cases}1 & (\varsigma>0) \\
i & (\varsigma<0),\end{cases}
\end{aligned}
$$

and

$$
\begin{aligned}
C & \approx 4 \int_{|q|>k} \frac{J_{1}(q \Delta)}{q \Delta}\left(\sqrt{q^{2}-k^{2}}\right) e^{i q x} d q \\
& \approx 4 k^{2}\left[\ln (\gamma k \Delta / 2)+\frac{2}{k^{2} \Delta^{2}}\right] .
\end{aligned}
$$
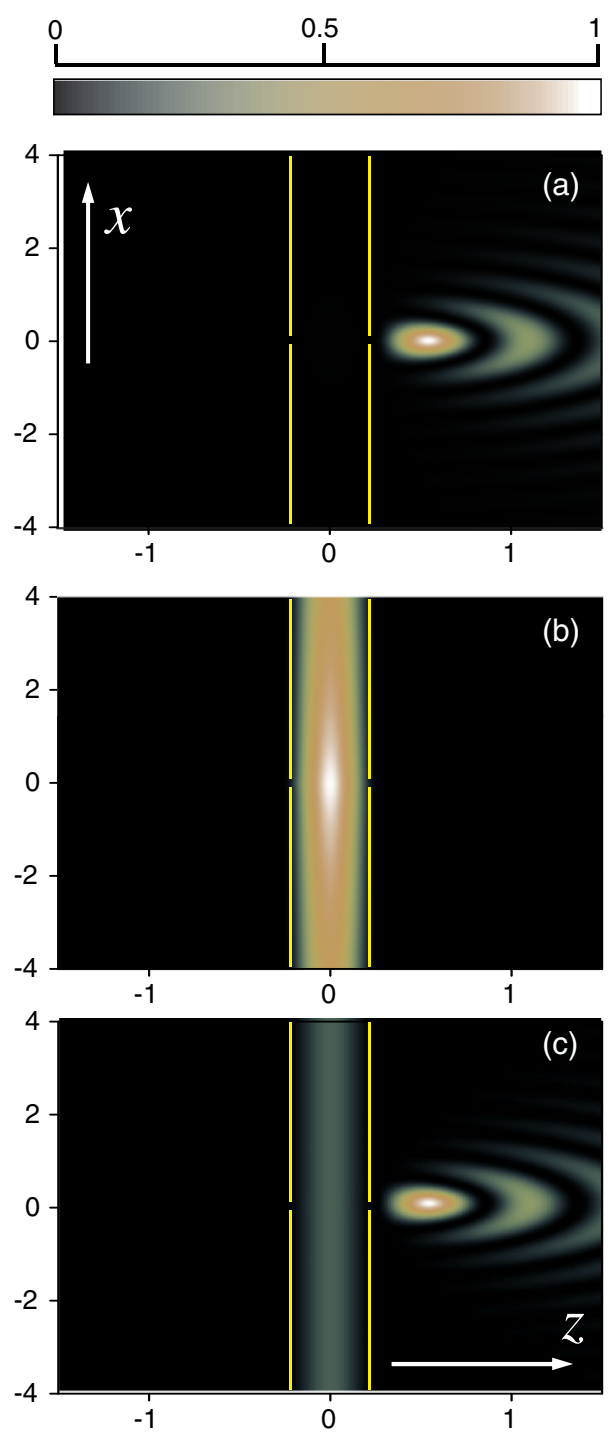

FIG. 2. Contour plots of $\left|E_{y}(x, z)\right|^{2}$ for $\Delta / \lambda=0.02\left(\varsigma_{R} \approx\right.$ $10^{-4}$ ), normalized to its largest value. Results are for $h=(\pi-\varsigma) / k$ with (a) $\mathrm{s}=2 \times 10^{2} \varsigma_{R}$, (b) $\mathrm{s}=\mathrm{s}_{R}$, and (c) $\mathrm{s}=2 \times 10^{-6} \mathrm{~s}_{R}$. The perfectly conducting screens are indicated by yellow lines. Cylindrical waves travel from right to left. Lengths are in units of $\lambda$.
Concerning the transmitted power, one can distinguish three regimes: (i) $C \gg B$ results in $0<\Gamma^{(-)} \ll$ $\Gamma^{(+)} \sim k \Delta^{2} Y_{0}$; (ii) $C=2 B$, that is,

$$
\mathrm{s}=\mathrm{s}_{\mathrm{R}} \approx \frac{\pi}{2}\left[\ln (\gamma k \Delta / 2)+\frac{2}{k^{2} \Delta^{2}}\right]^{-2},
$$

which gives $\Gamma^{(-)} \approx \Gamma^{(+)} \sim i \Upsilon_{0} / \pi k \quad\left(T_{N} \sim \lambda / \Delta\right)$; and (iii) $C \ll B$ with $\Gamma^{(-)} \approx-\Gamma^{(+)} \sim-k \Delta^{2} \Upsilon_{0}\left(T_{N} \sim \Delta^{3} / \lambda^{3}\right)$. The images in Fig. 2 show representative examples of the field intensity profile for the three cases. The features in Fig. 2(a) reflect mainly the spatial interference between the incident and reflected fields, i.e., $|Y|^{2}$, whereas the intensity pattern in Fig. 2(c) is that of the resonance with the lowest cutoff mode of the waveguide which, as shown later, manifests itself as a cusp in the transmittance. Transmission is negligible for (i); it is much larger and, surprisingly, comparable to that for a single slit in case (iii), and significantly larger for (ii). From Eq. (6), we can establish that the latter case is associated with a long-lived resonance centered at $\varsigma=\varsigma_{R}$, with quality factor $Q \sim \mathrm{s}_{R}^{-3 / 2} \sim(k \Delta)^{-6}$, that occurs at a frequency $\mathrm{s}_{R} \omega_{C} / \pi$ below the onset of the continuum. As suggested by the data of Fig. 2(b), the corresponding state acts like a cavity mode in the sense that it is localized and bound to the orifices inside the waveguide, but leaks weakly into free space through the narrow apertures. The existence of aperture-bound cavitylike modes between conducting plates holds promise for applications requiring both high spatial resolution and spectroscopic discrimination [25]. The localization length can be gained from Eq. (4). At $z=0,\left|E_{y}\right|^{2} \propto \exp (-x / \delta)$ with $\delta=\lambda / 4\left(\pi \varsigma_{R}\right)^{1 / 2}$. It can be further shown that this state is TE but not TM- active; that is, the fields do not couple to the localized resonance in the TM configuration.

\section{Resonant transmission: Localized (defect) state vs bulk cutoff mode}

We now show that the localized resonance leads to perfect transmission. At $\mathrm{s}=\mathrm{s}_{R}$,

$$
E_{y}(x, \pm h / 2)=\frac{2 i \Upsilon_{0}}{\pi k \Delta} \sqrt{1-x^{2} / \Delta^{2}} .
$$

Using the angular spectral decomposition [26], we obtain the following in the radiation zone $(\tilde{\rho} \gg \lambda)$ :

$$
E_{y}(x, z) \approx \frac{i \Upsilon_{0}}{2 \pi k} \int_{|q|<k} e^{i \sqrt{k^{2}-q^{2}}(z-h / 2)} e^{i q x} d q .
$$

Since the incident field can be written as

$$
\begin{aligned}
\Upsilon_{i} & =\Upsilon_{0} J_{1}(k \tilde{\rho}) \sin \tilde{\varphi} \\
& =\frac{\Upsilon_{0}}{2 i \pi k} \int_{|q|<k}\left(e^{i(z-h / 2) \sqrt{k^{2}-q^{2}}}-e^{-i(z-h / 2) \sqrt{k^{2}-q^{2}}}\right) e^{i q x} d q,
\end{aligned}
$$

it is clear that the emitted radiation exactly cancels the reflected beam. This effect is emphasized in Fig. 3 which shows $\left|E_{y}(x, z) / E_{y}(0, z)\right|^{2}$; the data are the same as in 


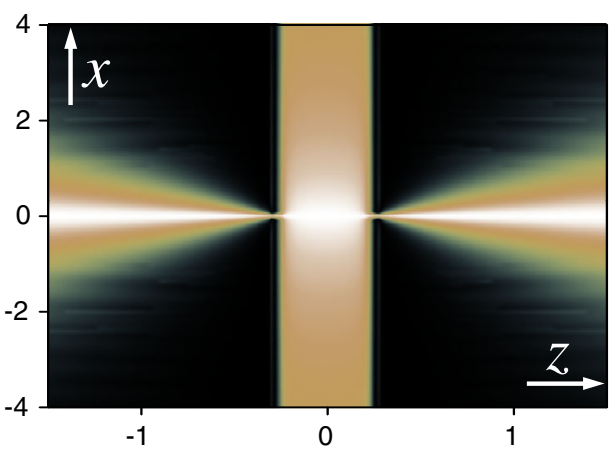

FIG. 3. Contour plot of $\left|E_{y}(x, z) / E_{y}(0, z)\right|^{2}$ for $\mathrm{s}=\mathrm{s}_{R}$. The values are the same as in Fig. 2(b).

Fig. 2(b). Note that such a normalization makes it appear that the intensity at $x=0$ is nearly the same regardless of the value of $z$; actually, the intensity at the origin is approximately $10^{3}$ times larger than at the openings.

As pointed out earlier in the discussion of case (iii), Fig. 2(c), the resonance with the extended cutoff mode does not give perfect transmission. Rather, the transmitted power is on the same order as that for a bare single slit. An inspection of work available in the literature shows that this also applies to the TM excitation of slotted plates [22] and, significantly, single-slit funneling, that is, TM transmission through a narrow slit carved in a metal slab $[8,11,21,27]$. Just as for our TE case, the power transmitted at the cutoff frequency $(k h=p \pi)$ in these two cases is compa- rable to that for off-resonance excitation of a single slit. Specifically, using expressions derived in $[11,12]$ we find that the common leading term for funneling and TM excitation of the slotted plates is

$$
T_{N} \propto \frac{\lambda / \Delta}{\ln ^{2}(\Delta / \lambda)},
$$

while, as already mentioned, the TE geometry gives $T_{N} \sim \Delta^{3} / \lambda^{3}$, exactly matching the results for single slits (note that the transmitted power vanishes for both configurations when $\Delta \rightarrow 0$ ). Parenthetically, we draw attention to the differences between funneling through individual slits and through slit arrays for which the slit separation is $d \ll \lambda$ [28]. Arrays behave as continuous anisotropic media described by effective permittivity and permeability tensors which, unlike individual slits (but like standard optical slabs), transmit perfectly TM-polarized plane waves at the Fabry-Pérot-like resonances [28].

It is apparent from the previous considerations that Eq. (2) correctly describes the transmission behavior of the localized resonance whereas, regardless of the geometry, it does not apply to bulk cutoff modes, which are extended. The example of TE excitation of slotted plates sheds light on the general relationship between cutoff resonances and single-slit transmission. From Eq. (4) at $k h \equiv \pi$, the difference between the field with and without the orifices $\delta E_{y}$ becomes simply

$$
\delta E_{y}(x, z)= \begin{cases} \pm a_{O} \int_{-\infty}^{+\infty} \frac{J_{1}(q \Delta)}{q \Delta} e^{i \kappa( \pm z-h / 2)} e^{i q x} d q & ( \pm z>h / 2) \\ a_{E} \cos k z+a_{O} \int_{-\infty}^{+\infty} \frac{J_{1}(q \Delta)}{q \Delta} \frac{\sin \kappa z}{\sin (\kappa h / 2)} e^{i q x} d q & (|z|<h / 2) .\end{cases}
$$

Here, $a_{E}$ and $a_{O}$ are constants representing the amplitude of the cutoff mode and that of the correction due to the presence of the apertures. After eliminating lower-order terms, the continuity of $\partial E_{y} / \partial z$ gives

$$
\begin{aligned}
& a_{E}=-\Upsilon_{0} / 4, \\
& a_{O} \approx \frac{h \Upsilon_{0}}{8 \pi}\left[\ln (\gamma \pi \Delta / 2 h)+\frac{2 h^{2}}{\pi^{2} \Delta^{2}}\right]^{-1} .
\end{aligned}
$$

The comparison with Eq. (5) shows that the expression for the odd term is exactly half that of $F(q)$ for an uncoupled single slit. Moreover, since the magnitude of $a_{E}$ is equal to half that of the incident field, the resulting fields acting on the two apertures have the same magnitude but opposite signs. Hence, the effect of the resonance is to bring up the amplitude of the cutoff mode so that the aperture that does not interact directly with the external field behaves as if it were in contact with a field half its value. Thus, the net transmission is formally identical to that of an individual slit. With minor modifications, it can be shown that these arguments apply as well to the two-slit TM problem and TM funneling.

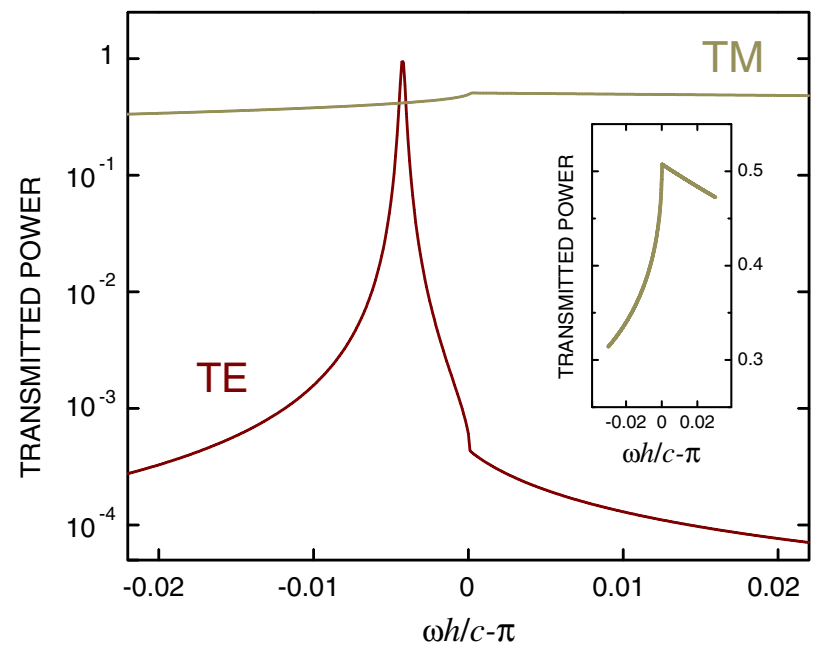

FIG. 4. Log plot of the transmitted power, normalized to the value corresponding to perfect transmission, as a function of the frequency. The data are for $\Delta=h / 10$. Results reported in [22] were used to get the TM curve. A linear plot for the TM geometry is shown in the inset. 
To underline the differences between the TE and TM geometries and recap the main results, we show in Fig. 4 the frequency dependence of the normalized transmittance in the vicinity of the fundamental Fabry-Pérot resonance. The TE and TM curves were obtained, respectively, from Eq. (6) and exact expressions derived in [22]. Both curves show a cusp at $\omega=\pi c / h$ and, as discussed above, the transmission at the cusps is on the order of that for a single unresonated slit [we stress the fact that the transmitted power for a single slit is a factor of approximate $\left(\lambda^{4} / \Delta^{4}\right) \ln ^{-2}(\Delta / \lambda)$ larger for the TM geometry]. As shown in the inset, the TM cusp corresponds to a maximum in the transmission. The TE spectrum exhibits not a maximum but a kink due to interference with the dominant resonance due to the localized mode.

\section{CONCLUSIONS}

In summary, we discussed a general model of wave transmission through apertures that accounts for the extraordinary properties of subwavelength openings that interact with a single oscillator. We also presented an analytically solvable example of perfect transmission associated with a state bound to openings cut in the walls of a two-dimensional waveguide, and showed that the resonance involving the extended cutoff mode of the bulk exhibits imperfect transmission properties, which are identical to those of a single, nonresonated aperture. Finally, we established that such a single-aperture-like behavior is a general property of resonances involving bulk modes, which do not interact directly with the incident field, and ascertained its relevance to the effect known as light funneling, that is, resonant transmission through subwavelength slits carved in a thick piece of metal.

\section{ACKNOWLEDGMENTS}

This work was supported in part by the Air Force Office of Scientific Research under Grant No. FA9550-09-1-0636 and by the MRSEC Program of the National Science Foundation under Grant No. DMR- 1120923. The author would like to thank J. Guo for introducing him to the problem of electromagnetic funneling, B. Isaacoff, M. Henstridge, and S. Young for help with the numerical calculations, and S. Fahy for suggesting the title.

[1] H. A. Bethe, Theory of Diffraction by Small Holes, Phys. Rev. 66, 163 (1944). Bethe's law, $T_{N} \sim \ell^{4} / \lambda^{4}$, applies to subwavelength holes in infinitesimally thin and perfectly conducting screens. Apertures in real screens, having finite conductivity and thickness, exhibit poorer transmission properties.

[2] C. J. Bouwkamp, Diffraction Theory, Rep. Prog. Phys. 17, 35 (1954).
[3] T. W. Ebbesen, H. J. Lezec, H. F. Ghaemi, T. Thio, and P. A. Wolff, Extraordinary Optical Transmission Through Subwavelength Hole Arrays, Nature (London) 391, 667 (1998).

[4] J.A. Porto, F. J. García-Vidal, and J. B. Pendry, Transmission Resonances on Metallic Gratings with Very Narrow Slits, Phys. Rev. Lett. 83, 2845 (1999).

[5] L. Martín-Moreno, F. J. García-Vidal, H. J. Lezec, T. Thio, J. B. Pendry, and T. W. Ebbesen, Theory of Extraordinary Optical Transmission through Subwavelength Hole Arrays, Phys. Rev. Lett. 86, 1114 (2001).

[6] F. J. García-Vidal, L. Martin-Moreno, T. W. Ebbesen, and L. Kuipers, Light Passing Through Subwavelength Apertures, Rev. Mod. Phys. 82, 729 (2010).

[7] C. Genet and T. W. Ebbesen, Light in Tiny Holes, Nature (London) 445, 39 (2007).

[8] R.F. Harrington and D. T. Auckland, Electromagnetic Transmission Through Narrow Slots in Thick Conducting Screens, IEEE Trans. Antennas Propag. 28, 616 (1980).

[9] R. F. Harrington, Resonant Behavior of a Small Aperture Backed by a Conducting Body, IEEE Trans. Antennas Propag. 30, 205 (1982).

[10] Y. Leviatan, R.F. Harrington, and J.R. Mautz, Electromagnetic Transmission Through Apertures in a Cavity in a Thick Conductor, IEEE Trans. Antennas Propag. 30, 1153 (1982).

[11] Y. Takakura, Optical Resonance in a Narrow Slit in a Thick Metallic Screen, Phys. Rev. Lett. 86, 5601 (2001).

[12] F. J. García-Vidal, H. J. Lezec, T. W. Ebbesen, and L. Martín-Moreno, Multiple Paths to Enhance Optical Transmission through a Single Subwavelength Slit, Phys. Rev. Lett. 90, 213901 (2003).

[13] X. Shi, L. Hesselink, and R. L. Thornton, Ultrahigh Light Transmission Through a C-Shaped Nanoaperture, Opt. Lett. 28, 1320 (2003).

[14] J. Wuenschell and H. K. Kim, Excitation and Propagation of Surface Plasmons in a Metallic Nanoslit Structure, IEEE Trans. Nanotechnol. 7, 229 (2008).

[15] F. Pardo, P. Bouchon, R. Haïdar, and J. L. Pelouard, Light Funneling Mechanism Explained by Magnetoelectric Interference, Phys. Rev. Lett. 107, 093902 (2011).

[16] L. Sahin, K. Aydin, G. T. Sayan, and E. Ozbay, Enhanced Transmission of Electromagnetic Waves Through SplitRing Resonator-Shaped Apertures, J. Nanophoton. 5, 051812 (2011).

[17] A mode is said to be localized if $\int U d \Sigma<\infty$, where $U$ is the electromagnetic energy density and $\Sigma$ is the volume or area. If, instead, the integral diverges, the state is extended.

[18] See, e.g., J. D. Jackson, Classical Electrodynamics (Wiley, New York, 1999), 3rd ed., p. 422.

[19] This result, applying to perfectly conducting screens of vanishing thickness, ignores contributions due to higherorder multipoles. As such, it is valid in the limit $\ell \ll \lambda$.

[20] P. Bharadwaj, B. Deutsch, and L. Novotny, Optical Antennas, Adv. Opt. Photon. 1, 438 (2009).

[21] F. Yang and J.R. Sambles, Resonant Transmission of Microwaves through a Narrow Metallic Slit, Phys. Rev. Lett. 89, 063901 (2002).

[22] Y. Leviatan, Electromagnetic Coupling Between Two HalfSpace Regions Separated by Two Slot-Perforated Parallel 
Conducting Screens, IEEE Trans. Microwave Theory Tech. 36, 44 (1988).

[23] C. M. Butler and D. R. Wilton, General Analysis of Narrow Strips and Slots, IEEE Trans. Antennas Propag. 28, 42 (1980).

[24] R.F. Harrington and J. R. Mautz, A Generalized Network Formulation for Aperture Problems, IEEE Trans. Antennas Propag. 24, 870 (1976).

[25] The localized TE mode is robust in that simulations using commercially available software indicate its persistence in structures made of real metals all the way into the optical range as well as for perfectly conducting screens with thickness much less than $\lambda$. Not unexpectedly, the transmission decreases strongly and the resonance becomes broader at shorter wavelengths due to increasing losses.

[26] G.C. Sherman, Integral-Transform Formulation of Diffraction Theory, J. Opt. Soc. Am. 57, 1490 (1967).

[27] S. Astilean, Ph. Lalanne, and M. Palamaru, Light Transmission Through Metallic Channels Much Smaller Than the Wavelength, Opt. Commun. 175, 265 (2000).

[28] F. J. García-Vidal, L. Martin-Moreno, and J. B. Pendry, Surfaces with Holes in Them: New Plasmonic Metamaterials, J. Opt. A 7, S97 (2005). 